\title{
Higher Groundwater Selenium Exposure Is Associated with Better Memory: A Project FRONTIER Study*
}

\author{
James Hall $^{1 \#}$, Melissa Edwards ${ }^{2}$, Robert Barber ${ }^{3}$, Leigh Johnson ${ }^{4}$, Gordon Gong ${ }^{5}$, Sid E. O’Bryant ${ }^{5,6}$ \\ ${ }^{1}$ Department of Psychiatry, University of North Texas Health Science Center, Fort Worth, USA; ${ }^{2}$ Department of Psychology, Texas \\ Tech University, Lubbock, USA; ${ }^{3}$ Department of Pharmacology and Neuroscience, University of North Texas Health Science Center, \\ Fort Worth, USA; ${ }^{4}$ Laura W. Bush Institute for Women's Health \& Department of Family and Community Medicine, Texas Tech \\ University Health Sciences Center, Lubbock, USA; ${ }^{5}$. Marie Hall Institute for Rural and Community Health, Texas Tech Health \\ Sciences Center, Lubbock, USA; ${ }^{6}$ Department of Neurology, Texas Tech University Health Sciences Center, Lubbock, USA. \\ Email: ${ }^{\#}$ James.Hall@unthsc.edu
}

Received December $12^{\text {th }}, 2011$; revised January $15^{\text {th }}, 2012$; accepted February $6^{\text {th }}, 2012$

\begin{abstract}
Background: Exposure to elements in groundwater (toxic or beneficial) is commonplace yet, outside of lead and mercury, little research has examined the impact of many commonly occurring exposures on mental abilities during the aging process. Selenium has antioxidant properties as part of the glutathione peroxidase system and may have protective effects on memory abilities. Objectives: To investigate the relationship of groundwater selenium exposure to neuropsychological status. Method: Analysis of data from 484 participants (148 men and 336 women) of Project FRONTIER, a community-based participatory research study of the epidemiology of health issues of rural-dwelling adults and elders. Results: Estimated selenium exposure (current and long-term) was specifically related to memory functioning without relation to other neurocognitive domains. The significant, positive link between selenium and memory (Immediate and Delayed) scores held regardless of APOE4 status as well as when the sample was restricted to only those without cognitive dysfunction. Current selenium was also associated with significantly reduced risk of cognitive decline prospectively. Conclusions: Higher selenium levels were associated with better memory functioning as well as reduced risk of cognitive decline among this community-based sample. Given the antioxidant properties of selenium, and the well-documented link between oxidative stress and the development of cognitive dysfunction and Alzheimer's disease, additional research is necessary to determine the utility of groundwater selenium monitoring as a potential population-wide prevention effort against Alzheimer's disease.
\end{abstract}

Keywords: Environmental Exposure; Cognition; APOE4; Selenium; Groundwater

\section{Introduction}

The link between environmental exposures through drinking water and health outcomes has received a great deal of attention. As an example, exposure to arsenic and lead through drinking water supplies has been associated to a range of poor health outcomes including cancer, cardiovascular disease, diabetes, and poor cognition [1-6]. Exposure to elements (toxic and non-toxic) through drinking water supplies is a common occurrence though not all of these elements have received adequate scientific attention.

Selenium is a trace element that has antioxidant properties through activity of glutathione peroxidase [7] and is commonly present in drinking water. Low and overly high levels of selenium have been associated with increased

\footnotetext{
*The authors have no conflict of interest to declare.

${ }^{\#}$ Corresponding author.
}

risk for a number of poor health conditions including cancer and cardiovascular disease $[7,8]$ and selenium levels have recently been found to be significantly lower among patients with Alzheimer's disease when compared to nondemented controls [9]. It has been suggested that cerebral oxidative stress is one of the leading causes of cognitive dysfunction [10] and a wealth of literature links oxidative stress and the development of cognitive dysfunction and/or dementia (i.e. Alzheimer's disease). Therefore, groundwater selenium exposure at optimum levels may hold promise as a potential preventative effort aimed at staving off or delaying onset of cognitive impairment at the population level. However, the link between selenium and neurocognitive status has only received minimal investigation in the literature [7,11-13]. Gao and colleagues [7] evaluated the link between selenium levels in fingernail samples and neuropsychological functioning using the CERAD neuropsychological battery in a sample of 2000 rural Chinese 
elders. In that study, lower selenium levels were significantly associated with poorer scores in memory and executive functioning, but not verbal fluency. On the other hand, Perkins et al. [13] failed to document a significant relationship between serum selenium levels and cognitive functioning when analyzing data from the National Health and Nutrition Examination project (NHANES). However, this discrepancy may have been due to the limited cognitive screening that was utilized in the NHANES. Longitudinally, Akbaraly et al. [11] analyzed data from a community-dwelling French elderly cohort and found that decreased plasma selenium level was associated with increased cognitive decline after nine years and the greater the decline in selenium over time, the greater chance of cognitive decline. The current study was undertaken to further this work by examining the link between groundwater selenium exposure and neuropsychological functioning.

The findings of a specific link between selenium and memory among elders by Gao et al. [7] is of critical importance since semantic memory deficits are among the first clinical manifestations of Alzheimer's disease. Given the rapidly growing epidemic of Alzheimer's disease worldwide [14] and the economic burden associated with this devastating disease [15], it is of critical importance that we understand factors that specifically impact memory functioning. Current therapeutics for Alzheimer's disease are only minimally efficacious, which has led many researchers to believe that prevention is the most appropriate strategy [16]. The recently highly publicized NIH panel discussion stated there are no current preventative strategies against this Alzheimer's disease; however, dietary factors were noted as promising avenues for further research. The identification of drinking water exposures that convey risk for or protection against memory decline and Alzheimer's disease would have profound implications as it could open up the possibility of population-based prevention strategies targeting this disease.

Therefore, the current study sought to build upon prior work by examining the link between groundwater selenium exposure and detailed neurocognitive functioning among a sample of rural-dwelling U.S. adults and elders. Based on the findings of Gao and colleagues [7], it was hypothesized that lower selenium levels would be specifically related to poorer memory scores. If groundwater selenium is to be useful in preventative efforts, it must also be related to memory functioning among those without overt signs of cognitive impairment. Therefore, we also hypothesized that selenium levels would be significantly related to memory performance when the sample was restricted to only those participants who were judged to be cognitively normal by consensus review. Gao et al. [17] in 2009 documented that fingernail levels of selenium were lower among $\varepsilon 4$ carriers of the APOE gene, which is also the strongest genetic risk factor for late-onset Alzheimer's disease. Therefore, we conducted exploratory analyses to determine if the link between selenium and cognitive functioning varied according to APOE $\varepsilon 4$ status. Additionally, it is likely that current exposure only provides partial picture of the importance of selenium given that one is exposed chronically over long periods of time through drinking water sources. Therefore, it was also hypothesized that long-term selenium exposure at current residence would be significantly positively related to memory abilities. Lastly, we conducted pilot analyses on the impact of selenium on change in global cognition over time by analyzing a sub-set of participants that were evaluated through an initial pilot study as well as through the current protocol. It was hypothesized that selenium levels would be significantly associated with less global cognitive decline.

\section{Methods}

Data was analyzed from 484 participants (148 men and 336 women) from Project FRONTIER. Project FRONTIER is an ongoing community-based participatory research (CBPR) study of health among rural-dwelling adults and elders. CBPR is a research methodology that partners scientific groups with community members and organizations to achieve the goals of the study. CBPR is particularly useful when working with underserved communities and it is recommended for rural research by the National Institute of Environmental Health Sciences [18]. In Project FRONTIER, we have spent several years establishing and maintaining community ties through advisory boards, presentations, community recruiters/assessors, and partnering with community entities for completion of parts of the research protocol (i.e. blood work and medical examinations). The findings from Project FRONTIER utilizing this approach have been previously published [19-22].

Once participants completed the consent process, s/he underwent an interview (participant and informant), standardized medical examination, clinical labs, and neuropsychological testing. Inclusion criteria were 1) age 40 and above and 2) residing in one of the counties part of Project FRONTIER. Data for the current study came from participants residing in Cochran County and Parmer County, both of which are located on the Texas New Mexico border. Participant recruitment was completed by community recruiters through multiple modalities including brochures/ flyers, presentations and events, in-person and/or door-todoor solicitation, as well as snowball recruitment. All participants signed written informed consent and Project FRONTIER is conducted under IRB approval.

In an initial pilot study, 316 participants in Cochran County were run through a brief protocol, which included a 
Mini-Mental State Examination (MMSE) to establish the feasibility of our CBPR approach. As of November 1, 2010, 188 of the original participants had completed the full Project FRONTIER protocol outlined above. Of the 188 participants, 135 had the requisite information available for the current study (i.e. baseline and follow-up MMSE scores and selenium estimates) and were included in the analyses ( 8 men and 127 women). The mean interval between testing was 1.8 years $(\mathrm{sd}=1.5)$.

The Repeatable Battery for the Assessment of Neuropsychological Status (RBANS). The RBANS [23] is a brief neuropsychological instrument that assesses multiple cognitive domains [24]. It contains 12 subtests that combine to create five indices: Attention, Language, Visuospatial/Constructional Skills, Immediate Memory and Delayed Memory. The RBANS has accumulated a large amount of normative data and has good psychometric properties and diagnostic accuracy [23, 25-27].

The Mini-Mental State Examination (MMSE) [28] is the most commonly administered psychometric screening assessment of global cognitive functioning. The MMSE assesses working memory, recall, orientation, naming, ability to follow commands, reading and writing and is used to screen for global cognitive impairment, track changes in cognitive functioning over time, and oftentimes to assess the effects of therapeutic agents on cognitive function [29]. Since its development, there has been a wealth of literature published on the MMSE demonstrating it to be a relatively sensitive marker of dementia [30].

Determination of GIS-Selenium. Geographic information system (GIS) is a way of displaying and analyzing geographically reference information. GIS-based methods are commonly used to estimate environmental exposures [31-35]. We used the Environmental Systems Research Institute (ESRI, 2009) ArcGIS (release 9.2) program to plot a point for each of the selenium ground water measurements from the publicly available Texas Water Development Board (TWDB). The TWDB is the most extensive state-level water surveillance system in the U.S. with over 14,000 data points going back over 15 years. Through inverse distance weighted (IDW) interpolation, the ArcGIS software builds a three dimensional surface map from a list of points. Each point's influence is weighted based off its distance to that section of the map, which was generated using 12 well measurements from the TWDB within the immediate geographic vicinity. Each of the study participant's current residential address was geocoded with the ArcGIS StreetMap data. Finally, GISselenium concentration was calculated by extracting the estimated selenium value from the IDW surface at each resident's location. The inter-rater reliability of our GIS approach to calculating our groundwater elements is very high (Pearson Correlation coefficient $>0.9$ ). In order to calculate an estimate of long-term selenium exposure (i.e. cumulative exposure at current residence), the number of years at current residence was multiplied by the current selenium estimates as the groundwater selenium in these regions has remained relatively consistent over the last $15+$ years.

APOE genotype $(\varepsilon 2, \varepsilon 3$, or $\varepsilon 4)$ was conducted on participants who provided whole blood for DNA extraction in the Project FRONTIER database. APOE allele genotyping was carried out via standardized PCR methods.

Using the entire sample, linear regression models were created using SPSS version 18 with raw RBANS index scores entered as outcome variables and selenium as predictor variables. Covariates entered into the models were age, gender, education, language of administration (English or Spanish) and APOE4 (presence/absence). In order to examine the link between chronic selenium exposure and cognition, the regression models described above were re-run using long-term selenium exposure estimates as the predictor variable. Lastly, to test the hypothesis that selenium (current and chronic) exposure would be significantly linked to memory functioning among normally functioning adults and elders, the sample was then restricted to only those participants who were judged to be "normal" on consensus review. Analyses were also re-run by APOE4 status (present/absent) to determine if the link between selenium and cognitive functioning varied according to this genetic marker. In order to determine if selenium exposure was related to change in global cognition, a MMSE change score was calculated

$(\triangle \mathrm{MMSE}=$ MMSEbaseline - MMSEfollowup). Next, a linear regression model was created with $\triangle \mathrm{MMSE}$ as the outcome variable and current selenium levels as the predictor variable; age, gender, education, and language of test administration were utilized as covariates.

Cognitive status was assigned by consensus panel according to the following criteria: normal cognition (no observed deficits on cognitive examination and no selfor informant-report of cognitive dysfunction-adapted from the Mayo criteria), Age Associated Cognitive Impairment (AACI) [36], Mild Cognitive Impairment (MCI) (without requirement of cognitive complaint) [37], Alzheimer's disease [38], vascular dementia [39], or dementia not otherwise specified (NOS, meets criteria for dementia but does not fit into one of the other categories).

\section{Results}

The mean age and education of the sample was 62.62 (sd $=12.75 ;$ range $=40-97)$ and $10.77(\mathrm{sd}=4.40 ;$ range $=0$ 20), respectively. Mean GIS-based selenium level was $18.91 \mu \mathrm{g} / \mathrm{L}(\mathrm{sd}=10.45$; range $=4.20-56.32)$. Seventyseven percent of the sample was tested in English with the remainder completing the assessment in Spanish. Eighty-eight percent of the sample self-reported their race as White and 38\% reported their ethnicity as Mexi- 
can American. Demographic characteristics of the sample are presented in Table 1. Consensus classification of participants regarding cognitive status was as follows: normal $\mathrm{n}=324$, AACI $\mathrm{n}=49$, MCI $\mathrm{n}=96$ and dementia (all cause) $n=15$. On average, participants resided in their current residence for 34 years $(\mathrm{sd}=20 \mathrm{yr}$, range $=1$ $80 \mathrm{yr}$ ). Of those who had been genotyped, there were 338 participants (74\%) who were APOE4 negative and 117 (26\%) who were APOE4 positive. Table 1 presents the demographic characteristics of the sample along with mean selenium levels and mean RBANS scores.

In the subset seen longitudinally from the pilot project $(\mathrm{n}=135)$, baseline mean age and MMSE scores were $60.7(\mathrm{sd}=13.4$, range $=40-94)$ and $27.3(\mathrm{sd}=2.5$, range $=18-30)$, respectively. Mean $\triangle$ MMSE was 0.35 $(\mathrm{sd}=2.30$; range $=7-13)$. Of note, a negative score is reflective of an increased MMSE score over time periods; therefore, on average, participants declined approximately one-half point on the MMSE over time though some declined a total of 13 points.

In the total sample, selenium levels were not significantly related to global cognition (i.e. MMSE scores) ( $p$ $>0.05$ ). Selenium levels were significantly and positively related to RBANS memory indices. Specifically, higher selenium was significantly related to better scores in Immediate Memory $(\beta=0.107, \mathrm{p}=0.01)$ and Delayed Memory, $(\beta=0.325, \mathrm{p}<0.001)$. Selenium levels were not related to other cognitive domains (i.e. Attention, Visouspatial skills, and Language). The results of the regression analysis can be found on Table 2. Current selenium exposure was significantly related to Immediate Memory among APOE4 ne-

Table 1. Demographic characteristics.

\begin{tabular}{ccc}
\hline & Mean (sd) & Range \\
Age & $62.62(12.75)$ & $40-97$ \\
Education & $10.77(4.40)$ & $0-20$ \\
GIS-selenium ( $\mu$ g/L) & $18.91(10.45)$ & $4.20-56.32$ \\
RBANS Immediate Memory & & \\
40.89 (9.47) 5 - 61 & & \\
RBANS Visuospatial & $28.90(6.36)$ & $8-40$ \\
RBANS Language & $27.05(5.53)$ & $9-100$ \\
RBANS Attention & & $10-60$ \\
RBANS Delayed Memory & & \\
36.53 (9.21) 10 - 60 & $36.53(9.21)$ & \\
\hline
\end{tabular}

$\mathrm{sd}=$ standard deviation; GIS = Geographic Information System; RBANS = Repeatable Battery for the Assessment of Neuropsychological Status. gative participants $(\beta=0.105, \mathrm{p}=0.037)$, but not APOE4 positive individuals. Selenium was significantly, positively associated with Delayed Memory scores among both APOE4 positive $(\beta=0.289, \mathrm{p}=0.003)$ and APOE4 negative $(\beta=0.341, \mathrm{p}<0.001)$ individuals.

Long-term exposure to selenium over the number of years at the current residence was only significantly positively related to Delayed Memory $(\beta=0.268, \mathrm{p}<0.001)$ without being related to other neuropsychological domains. This finding held for both APOE4 positive $(\beta=0.273, \mathrm{p}$ $=0.02)$ and APOE4 negative $(\beta=0.226, \mathrm{p}=0.001)$ participants.

In order to test the hypotheses that selenium (current and long-term) would be associated with better memory scores among cognitively normal individuals, the analyses were re-run utilizing only those consensus classified as "cognitively normal." In those without any overt signs of cognitive dysfunction, selenium level was again significantly positively associated with Immediate Memory $(\beta=0.23, \mathrm{p}<0.001)$ and Delayed Memory $(\beta=0.45, \mathrm{p}=$ $<0.001)$. As with the findings from the total sample, the positive link between current selenium exposure and Immediate Memory only held for APOE4 negative participants $(\beta=0.250, \mathrm{p}<0.001)$ while the link for Delayed Memory held for both APOE4 positive $(\beta=0.425, \mathrm{p}=0.001)$ and APOE4 negative $(\beta=0.472, \mathrm{p}<0.001)$ participants. Long-term selenium was again significantly positively related to Delayed Memory only $(\beta=0.37, \mathrm{p}<0.001)$, which held for both APOE4 positive $(\beta=0.410, \mathrm{p}=0.01)$ and APOE4 negative $(\beta=0.366, \mathrm{p}<0.001)$ participants.

As with the larger sample, current and long-term sele

Table 2. Selenium levels impact on neuropsychological functioning.

\begin{tabular}{ccccc}
\hline & Total Sample & \multicolumn{2}{c}{ Cognitively Normal $)$} \\
\hline & $(\mathrm{n}=324)$ & $(\mathrm{n}=484)$ \\
\hline & $\mathrm{B}(\mathrm{SE})$ & $\mathrm{p}$-value & $\mathrm{B}(\mathrm{SE})$ & $\mathrm{p}$-value \\
RBANS & $0.094(0.04)$ & 0.01 & $0.149(0.03)$ & $<0.001$ \\
$\begin{array}{c}\text { Immediate Memory } \\
\text { RBANS } \\
\text { Visuospatial }\end{array}$ & $0.027(0.03)$ & 0.30 & $0.020(0.03)$ & 0.53 \\
$\begin{array}{c}\text { RBANS } \\
\text { Language }\end{array}$ & $-0.038(0.02)$ & 0.08 & $-0.017(0.02)$ & 0.47 \\
$\begin{array}{c}\text { RBANS } \\
\text { Attention }\end{array}$ & $-0.079(0.08)$ & 0.13 & $0.011(0.06)$ & 0.84 \\
$\begin{array}{c}\text { RBANS } \\
\text { Delayed Memory }\end{array}$ & $0.281(0.04)$ & $<0.001$ & $0.305(0.04)$ & $<0.001$ \\
\hline B & & & & \\
\hline
\end{tabular}

$\mathrm{B}=$ Unstandardized Coefficient; SE = Standard Error Note. Covariates included age, gender, education, language of administration (English or Spanish), and APOE4 presence (yes/no). 
nium were not significantly related to baseline MMSE scores. However, current selenium was significantly related to a reduced risk of decline in MMSE scores over time $(\beta=-0.20, p=0.03)$. In fact, once current selenium levels were entered into the model, none of the other covariates (including age, gender, education and language of administration) contributed significantly to change in scores. Long-term selenium was not significantly related to $\triangle \mathrm{MMSE}$ scores.

\section{Discussion}

Water is consumed by everyone to varying degrees through direct consumption, cooking, bathing and/or personal hygiene (e.g. brushing teeth), recreational activities and many other ways. Identification of elements through this wide ranging exposure can positively or negatively impact mental status during the aging process would have profound implications for public health. Our findings suggest that groundwater selenium is an important element to consider. Others have proposed the use of selenium for preventative and/or treatment efforts aimed at cognitive dysfunction and Alzheimer's disease.

The current findings cross-validate prior work that has linked selenium specifically to memory functioning. Consistent with the work of Gao et al. [7], analysis of our sample of 484 rural-dwelling adults and elders, revealed that lower selenium level was significantly related to poorer memory among the total sample as well as when restricted to only those considered cognitively normal. Through use of GIS methods, we also extend this work to a new modality of measurement. Specifically, while Gao et al. found a significant link between memory and selenium levels in fingernails, we cross-validated this finding utilizing GIS-based estimated groundwater exposure to selenium. While inclusion of direct measurement of selenium levels in groundwater sources is preferable, GIS-based estimates such as those provided here enable a broader approach to this important area of study and open up inquiry to existing cohorts/databases of cognitive aging where groundwater selenium levels are available from other sources of data. The use of GIS methods also offers the distinct advantage of modeling estimated long-term exposure. Currently there is no biomarker of long-term selenium exposure available; however, the state of Texas has a longstanding water surveillance system through the Texas Water Development Board. With this historical data in hand, we first determined that groundwater selenium levels have been fairly stable over the last 15 years. We then were able to create a long-term estimate by multiplying current levels by the number of years residing at the participant's current home. Given the significant stability of the cohort (averaging over 30 years in current home), we were able to create the first ever estimate of long-term selenium exposure and demonstrate its link with memory abilities. Our preliminary analyses from a subset of participants seen prospectively $(\mathrm{n}=135)$, suggest that selenium exposure is related to less decline in global cognition over time. This finding is very interesting given that baseline MMSE scores were not related to selenium levels (current or longterm). These findings further support the need for further work looking at selenium as a preventative effort aimed at staving off or preventing cognitive decline.

The protective impact of selenium on memory functioning fits its biological activity. Selenium, a main constituent of neuronal selenoproteins, may be particularly important for sustaining healthy cerebral functioning [40] given the selenium dependent glutathione peroxidase line of defense against free radical damage [41]. An additional protective aspect of selenopritein is through selenoprotein $\mathrm{P}$, which is synthesized at the cerebral level and protects against oxidative damage due to peroxynitrite $[42,43]$. In animal models, short-term supplementation of selenium has been found to improve memory functioning in a rat model of dementia [44]. van Eersel et al. [45] demonstrated that supplementation of sodium selenate reduced tau phosphorylation and abrogated neurofibulary tangle (NFT) formation in multiple mouse models of Alzheimer's disease. In humans, low selenium levels have been associated with poorer memory functioning in crosssectional research [7] and with greater cognitive decline in longitudinal studies [11]. The current findings support both the cross-sectional and longitudinal benefit of higher selenium levels.

There are limitations to the current study worth considering. First, the use of direct ground-water measurement would be preferable. However, prior work has shown that GIS-based methods are valid for estimating current exposure levels. GIS-based methods also open up this line of research to additional research groups who may have neuropsychological data among large cohorts (cross-sectional and longitudinal), but do not have groundwater selenium levels if such records are available by other sources. The existence of the TWDB data showing stability of selenium groundwater levels over time, combined with the stability of the cohort, also offer the first ever method for attempting to model long-term selenium exposure. While we cannot conclude that the current findings are directly related to drinking water levels (we have estimated exposure levels rather than actual groundwater levels and consumption patterns), we can conclude that individuals who have resided in regions of lower groundwater selenium levels (current and over long periods of time) have poorer memory than those living in areas of higher selenium groundwater levels. The current findings do not take into account selenium ingestion from other sources (i.e. food, supplementation). We do not have detained neuropsychological test findings longitudinally; however, our pilot analyses on change in MMSE scores 
over time support the need to further investigate this topic and we will begin collection of longitudinal data in Project FRONTIER soon.

Selenium has long been known to have anti-oxidant impact in the human body as well as within the brain, particularly. Oxidative stress is also one of the primary factors associated with age-related cognitive decline and dysfunction; however, little research has been conducted within human populations examining the potential positive impact of selenium on cognition. Our work adds to the extant literature by demonstrating a positive link between groundwater selenium exposure levels and memory abilities among a community-based sample of rural adults and elders. That link held for the total sample, as well as when restricted to those without any overt cognitive dysfunction and the link was only minimally impacted by APOE4 status. Our findings also support the link between selenium exposure and reduced decline in cognition longitudinally. If supported through additional investigation, these findings point to the potential of groundwater supplementation with selenium, within a therapeutic window, as a population-based strategy for decreasing risk for cognitive decline and/or Alzheimer's disease.

\section{Acknowledgements}

This work was funded in part by the Science to Achieve Results (STAR) Program of the U.S. Environmental Protection Agency (EPA) grant \# RD83479401 (O’Bryant, PI).

\section{REFERENCES}

[1] K. Bandeen-Roche, T. A. Glass, K. I. Bolla, A. C. Todd and B. S. Schwartz, "Cumulative Lead Dose and Cognitive Function in Older Adults," Epidemiology, Vol. 20, No. 6, 2009, pp. 831-839. doi:10.1097/EDE.0b013e3181b5f100

[2] C. Liao, H. H. Shen, C. L. Chen, L. I. Hsu, T. L. Lin, S. C. Chen and C. J. Chen, "Risk Assessment of Arsenic-Induced Internal Cancer at Long-Term Low Dose Exposure," Journal of Hazardous Materials, Vol. 165, No. 1-3, 2009, pp. 652-663. doi:10.1016/j.jhazmat.2008.10.095

[3] A. Navas-Acien, E. K. Silbergeld, R. Pastor-Barriuso, et al., "Arsenic Exposure and Prevalence of Type 2 Diabetes in US Adults," JAMA, Vol. 300, No. 7, 2008, pp. 814822. doi:10.1001/jama.300.7.814

[4] Arsenic in Drinking Water, Subcommittee on Arsenic in Drinking Water National Research Council, Washington DC, 1999.

[5] Arsenic in Drinking Water, Subcommittee to Update the 1999 Arsenic in Drinking Water Report National Research Council, 2001 Update, Washington DC, 2001.

[6] J. Weuve, S. A. Korrick, M. A. Weisskopf, L. M. Ryan, J. Schwartz, H. Nie, et al., "Cumulative Exposure to Lead in Relation to Cognitive Function in Older Women," Environmental Health Perspectives, Vol. 117, No. 4, 2009, pp. 574-580.
[7] S. Gao, Y. Jin, K. S. Hall, C. Liang, F. W. Unverzagt, R. $\mathrm{Ji}$, et al., "Selenium Level and Cognitive Function in Rural Elderly Chinese," American Journal of Epidemiology, Vol. 165, No. 8, 2007, pp. 955-965. doi:10.1093/aje/kwk073

[8] A. Flatt, N. Pearce, C. D. Thomson, M. R. Sears, M. F. Robinson and R. Beasley, "Reduced Selenium in Asthmatic Subjects in New Zealand," Thorax, Vol. 45, No. 2, 1990, pp. 95-99. doi:10.1136/thx.45.2.95

[9] B. R. Cardoso, T. P. Ong, W. Jacob-Filho, O. Jaluul, M. I. D. Freitas and S. M. F. Cozzolino, "Nutritional Status of Selenium in Alzheimer's Disease Patients," British Journal of Nutrition, Vol. 103, No. 6, 2010, pp. 803-806. doi:10.1017/S0007114509992832

[10] T. Finkel and N. J. Holbrook, "Oxidants, Oxidative Stress and the Biology of Ageing," Nature, Vol. 408, 2000, pp. 239-247. doi:10.1038/35041687

[11] N. T. Akbaraly, I. Hininger-Favier, I. Carrière, J. Arnaud, V. Gourlet, A. M. Roussel, et al., "Plasma Selenium over Time and Cognitive Decline in the Elderly," Epidemiology, Vol. 18, No. 1, 2007, pp. 52-58. doi:10.1097/01.ede.0000248202.83695.4e

[12] A. B. Mendelsohn, S. H. Belle, G. P. Stoehr and M. Ganguli, "Use of Antioxidant Supplements and Its Association with Cognitive Function in a Rural Elderly Cohort: The Movies Project," American Journal of Epidemiology, Vol. 148, No. 1, 1998, pp. 38-44. doi:10.1016/j.jalz.2008.02.005

[13] A. J. Perkins, H. C. Hendrie, C. M. Callahan, S. Gao, F. W. Unverzagt, Y. Xu, et al., "Association of Antioxidants with Memory in a Multiethnic Elderly Sample Using the Third National Health and Nutrition Examination Survey," American Journal of Epidemiology, Vol. 150, No. 1, 1999, pp. 37-44.

[14] Alzheimer's Association, “Alzheimer's Disease Facts and Figures," Alzheimer's \& Dementia, Vol. 4, No. 2, 2008, pp. 110-133.

[15] Alzheimer's Association, "Changing the Trajectory of Alzheimer's Disease," Washington DC, 2010.

[16] N. R. Graff-Radford, J. E. Crook, J. Lucas, B. F. Boeve, D. S. Knopman, R. J. Ivnik, et al., "Association of Low Plasma Abeta42/Abeta40 Ratios with Increased Imminent Risk for Mild Cognitive Impairment and Alzheimer Disease," Archives of Neurology, Vol. 64, No. 3, 2007, pp. 354-362. doi:10.1001/archneur.64.3.354

[17] S. Gao, Y. Jin, K. S. Hall, C. Liang, F. W. Unverzagt, F. Ma, et al., "Selenium Level Is Associated with apoE $\varepsilon 4$ in Rural Elderly Chinese," Public Health Nutrition, Vol. 12, No. 12, 2009, pp. 2371-2376. doi:10.1017/S1368980009005102

[18] L. D. O'Fallon, “Commitment of the National Institute of Environmental Health Sciences to Community-Based Participatory Research for Rural Health," Environmental Health Perspectives, Vol. 109, Suppl. 3 2001, pp. 469473.

[19] L. Johnson, V. Hobson, M. Jenkins, A. Dentino, M. Ragain and S. E. O'Bryant, "The Influence of Thyroid Function on Cognition in a Sample of Ethnically Diverse, Rural- 
Dwelling Women: A Project FRONTIER Study," Journal of Neuropsychiatry and Clinical Neuroscience, Vol. 23, No. 2, 2011, pp. 219-222. doi:10.1176/appi.neuropsych.23.2.219

[20] S. E. O’Bryant, Y. Zhang, D. Owen, B. Cherry, V. Ramirez, M. Silva, C. Hudson, V. Hobson, P. Grammas, R. B. Schiffer, G. Manning, G. W. Schrimsher, J. A. Lucas, P. B. Sutker and the CCAS Research Team, "The Cochran County Aging Study: Methodology and Descriptive Statistics," Texas Public Health Journal, Vol. 61, No. 1, 2009, pp. 5-7.

[21] S. E. O'Bryant, J. Falkowski, V. Hobson, L. Johnson, J. Hall, G. W. Schrimsher, et al., "Executive Functioning Mediates the Link between Other Neuropsychological Domains and Daily Functioning: A Project FRONTIER Study," International Psychogeriatrics, Vol. 23, No. 1, 2010, pp. 107-113. doi:10.1017/S1041610210000967

[22] S. O’Bryant, J. R. Hall, K. Cukrowizc, M. Edwards, L. A. Johnson, D. Lefforge, M. Jenkins and A. Dentino, "The Differential Impact of Depressive Symptom Clusters on Cognition in a Rural Multi-Ethnic Cohort: A Project FRONTIER Study," International Journal of Geriatric Psychiatry, Vol. 26, No. 2, 2011, pp. 199-205. doi:10.1002/gps.2514

[23] C. Randolph, "Repeatable Battery for the Assessment of Neuropsychological Status," The Psychological Coporation, San Antonio, 1998.

[24] K. Duff, L. J. Beglinger, M. R. Schoenberg, D. E. Patton, J. Mold, J. G. Scott, et al., "Test-Retest Stability and Practice Effects of the RBANS in a Community Dwelling Elderly Sample," Journal of Clinical and Experimental Neuropsychology, Vol. 27, No. 5, 2005, pp. 565-575. doi:10.1080/13803390490918363

[25] K. Duff, D. Pattern, M. R. Schoenberg, J. Mold, J. G. Scott and R. L. Adams, "Age- and Education-Corrected Independent Normative Data for the RBANS in a Community Dwelling Elderly Sample," Clinical Neuropsychologist, Vol. 17, No. 3, 2003, pp. 351-366. doi:10.1076/clin.17.3.351.18082

[26] K. Duff, J. D. Humphreys, S. E. O’Bryant, J. W. Mold, R. B. Schiffer and P. B. Sutker, "Utility of the RBANS in Detecting Cognitive Impairment Associated with Alzheimer's Disease: Sensitivity, Specificity, and Positive and Negative Predictive Powers," Archives of Clinical Neuropsychology, Vol. 23, No. 5, 2008, pp. 603-612. doi:10.1016/j.acn.2008.06.004

[27] E. B. Larson, K. Kirschner, R. Bode, A. Heinemann and R. Goodman, "Construct and Predictive Validity of the Repeatable Battery for the Assessment of Neuropsychological Status in the Evaluation of Stroke Patients," Journal of Clinical \& Experimental Neuropsychology, Vol. 27, No. 1, 2005, pp. 16-32. doi:10.1080/138033990513564

[28] M. F. Folstein, S. E. Folstein and P. R. McHugh, "MiniMental State: A Practical Method for Grading the Cognitive State of Patients for the Clinician," Journal of Psychiatric Research, Vol. 12, No. 3, 1975, pp. 189-198. doi:10.1016/0022-3956(75)90026-6

[29] E. Strauss, E. M. S. Sherman and O. Spreen, "A Com- pendium of Neuropsychological Tests: Administration, Norms, and Commentary," 3rd Edition, Oxford University Press, Oxford, 2006.

[30] J. R. Harvan and V. Cotter, "An Evaluation of Dementia Screening in the Primary Care Setting," Journal of the American Academy of Nurse Practitioners, Vol. 18, No. 8, 2006, pp. 351-360. doi:10.1111/j.1745-7599.2006.00137.x

[31] G. A. AvRuskin, G. M. Jacquez, J. R. Meliker, M. J. Slotnick, A. M. Kaufmann and J. O. Nriagu, "Visualization and Exploratory Analysis of Epidemiologic Data Using a Novel Space Time Information System," International Journal of Health Geographics, Vol. 3, 2004, p. 26. doi:10.1186/1476-072X-3-26

[32] G. Gong and S. E. O'Bryant, "The Arsenic Exposure Hypothesis for Alzheimer's Disease," Alzheimer Disease \& Associated Disorders, 2010, Epub ahead of print.

[33] N. I. Khan, G. Owens, D. Bruce and R. Naidu, "Human Arsenic Exposure and Risk Assessment at the Landscape Level: A Review," Environmental Geochemistry and Health, Vol. 31, No. 1, 2009, pp. 143-166. doi:10.1007/s10653-008-9240-3

[34] S. R. Samadder and C. Subbarao, "GIS Approach of Delineation and Risk Assessment of Areas Affected by Arsenic Pollution in Drinking Water," Journal of Environmental Engineering, Vol. 133, No. 7, 2007, pp. 742-749. doi:10.1061/(ASCE)0733-9372(2007)133:7(742)

[35] C. C. Su, Y. Y. Lin, T. K. Chang, C. T. Chiang, J. A. Chung, Y. Y. Hsu, et al., "Incidence of Oral Cancer in Relation to Nickel and Arsenic Concentrations in Farm Soils of Patients' Residential Areas in Taiwan," $B M C$ Public Health, Vol. 10, 2010, p. 10.

[36] T. Crook, R. T. Bartus, S. H. Ferris, et al., "Age-Associated Memory Impairment: Proposed Diagnostic Criteria and Measures of Clinical Change: Report of a National Institute of Mental Health Work Group," Developmental Neuropsychology, Vol. 2, No. 4, 1986, pp. 261-276. doi: $10.1080 / 87565648609540348$

[37] R. C. Petersen, "Mild Cognitive Impairment: Aging to Alzheimer's Disease," Oxford University Press, Oxford, 2003.

[38] D. McKhann, D. Drockman, M. Folstein, et al., "Clinical Diagnosis of Alzheimer's Disease: Report of the NINCDSADRDA Work Group," Neurology, Vol. 34, No. 7, 1984, pp. 939-944.

[39] H. C. Chui, J. I. Victoroff, D. Margolin, W. Jagust, R. Shankle and R. Katzman, "Criteria for the Diagnosis of Ischemic Vascular Dementia Proposed by the State of California Alzheimer's Disease Diagnostic and Treatment Centers,” Neurology, Vol. 42, No. 3, 1992, pp. 473-480.

[40] J. Chen and M. J. Berry, "Selenium and Selenoproteins in the Brain and Brain Diseases," Journal of Neurochemistry, Vol. 86, No. 1, 2003, pp. 1-12. doi:10.1046/j.1471-4159.2003.01854.x

[41] M. P. Rayman, "The Importance of Selenium to Human Health," Lancet, Vol. 356, No. 9225, 2000, pp. 233-241. doi:10.1016/S0140-6736(00)02490-9

[42] U. Schweizer, A. U. Bräuer, J. Köhrle, R. Nitsch, N. E. 
Savaskan, "Selenium and Brain Function: A Poorly Recognized Liaison," Brain Research Reviews, Vol. 45, No. 3, 2004, pp. 164-178.

doi:10.1016/j.brainresrev.2004.03.004

[43] V. Mostert and P. Selenoprotein, "Properties, Functions, and Regulation," Archives of Biochemistry and Biophysics, Vol. 376, No. 2, 2000, pp. 433-438.

doi:10.1006/abbi.2000.1735

[44] T. Ishrat, K. Parveen, M. M. Khan, G. Khuwaja, M. B. Khan, S. Yousuf, et al., "Selenium Prevents Cognitive Decline and Oxidative Damage in Rat Model of Strepto-
zotocin-Induced Experimental Dementia of Alzheimer's Type," Brain Research, Vol. 1281, 2009, pp. 117-127. doi:10.1016/j.brainres.2009.04.010

[45] J. Van Eersel, Y. D. Ke, X. Liu, F. Delerue, J. J. Kril, J. Götz, et al., "Sodium Selenate Mitigates Tau Pathology, Neurodegeneration, and Functional Deficits in Alzheimer's Disease Models," Proceedings of the National Academy of Sciences of the United States of America, Vol. 107, No. 31, 2010, pp. 13888-13893.

doi:10.1073/pnas.1009038107 